\title{
Manuel Llorca-Jaña y Rory M. Miller (2021). Historia Económica de Chile desde la Independencia. Santiago de Chile: RIL Editores.
}

\author{
Ignacio González-Correa ${ }^{1,}$ * (DD 0000-0003-0964-8017 \\ ${ }^{1}$ Universidad de Santiago de Chile, Departamento de Estudios Políticos, Chile. \\ *Correspondencia: ignacio.gonzalez@usach.cl
}

Historia Económica de Chile desde la Independencia es una lectura recomendada ampliamente para las personas que estén interesadas en la historia de Chile y, en especial, en su historia económica. Se trata del primer manual de esta disciplina, el cual se une a Capitalists, Business and State-Building in Chile (2019), también con autoría de Manuel Llorca-Jaña y Rory Miller (editado junto a Diego Barría) y los libros editados en español de Manuel Llorca-Jaña y Diego Barría: Empresas y Empresarios en la Historia de Chile, 1810-1930 (2017) y Empresas y Empresarios en la Historia de Chile, 1930-2015 (2018).

Historia Económica de Chile desde la Independencia contiene poco más de 850 páginas y está organizado en 21 capítulos, además de un prólogo de Marcello Carmagnani. El manual cuenta con tres secciones: 1) la periodización ortodoxa; 2) los trabajos sectoriales de largo plazo, y 3) capítulos temáticos (misceláneos). Se trata de una obra destinada a estudiantes de pregrado y posgrado en las ciencias históricas y económicas. Para estos últimos, se vuelve relevante pues varias universidades chilenas cuentan con cursos obligatorios y optativos de historia económica y, hasta el momento, no existía un manual para abordar dicha materia. Además, también es un libro apto para el público general, ya que la mayoría de los capítulos están escritos en un lenguaje ameno y el uso de matemáticas o modelos estadísticos elaborados están acotados a la bibliografía.

Asimismo, en la introducción del libro se exponen una serie de trabajos "fundacionales" de la disciplina en Chile, mismos que han sido predecesores de este manual. Algunos casos notables son el libro de Aníbal Pinto Santa Cruz, Chile, un caso de desarrollo frustrado (1959); la obra de Arnold J. Bauer, Chilean Rural Society: From the Spanish Conquest to 1930 (1975, o su versión revisada
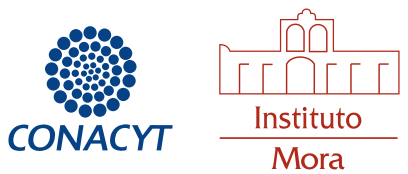
y en español del año 1994), o la obra de Carmen Cariola y Osvaldo Sunkel, Un siglo de historia económica de Chile, 1830-1930: dos ensayos y una bibliografía (1982), solo por mencionar algunos. Dichos trabajos permiten que los lectores conozcan cuáles fueron los cimientos sobre los que se ha construido la historia económica chilena.

Respecto a su composición, con carácter introductorio, el primer capítulo expone la estructura del libro, su relevancia y la síntesis de los siguientes apartados. Luego, en la primera parte del libro (desde el capítulo segundo al octavo), distintos autores nos muestran la historia económica de Chile caracterizada por la división de etapas o eras tradicionales: la temprana república (c. 1810-1879); la era del salitre (1880-1930); el periodo comprendido desde la Gran Depresión hasta la segunda guerra mundial (c. 1930-1947); la crisis del modelo de desarrollo y las reformas estructurales (1952-1970); la Unidad Popular (1970-1973); el modelo económico de la dictadura militar (c. 1973-1989), y la economía chilena desde el regreso a la democracia (1990 al presente).

La segunda parte del manual, desde el capítulo 9 al 15, corresponde al análisis de largo plazo por sectores claves: agricultura, minería, política comercial, dinero y banca, infraestructura y transportes, energía y desarrollo industrial. Estos capítulos son muy interesantes para el estudio por sector económico, sobre todo porque Chile se ha caracterizado por ser una economía basada en alta explotación (pero poca variedad) de recursos primarios. En adición, se exponen los principales debates y evidencia sobre el desempeño y el desarrollo de los distintos sectores económicos. Así pues, esta sección nos muestra cómo Chile cambió su forma de realizar la actividad económica, que pasó de estar basada principalmente en el sector agropecuario y minero en la época colonial, transitó en su vida republicana por la introducción al capitalismo para luego pasar por el modelo de industrialización con intervención estatal, hasta llegar a la adopción del neoliberalismo en el último cuarto del siglo xx.

La tercera sección de la obra posee una naturaleza miscelánea, comprende desde el capítulo 16 hasta el 21, en esta sección se abordan temáticas como empresarios y grupos económicos, evolución demográfica, trayectorias de salarios y precios, distribución del ingreso en el largo plazo (muy relevante luego del "estallido social" de octubre de 2019), la economía mapuche y la economía colonial. Acá, el horizonte temporal es difuso entre capítulos, la mayoría de los ensayos se concentran en la época de la república independiente, aunque hay un ensayo sobre el periodo colonial y otro que atraviesa ambos periodos (véase el capítulo de la economía mapuche). En esta sección se presentan quizás los trabajos más interesantes del libro: la economía mapuche, la distribución del ingreso y la historia empresarial.

En su conjunto, el enfoque del libro es más cualitativo que cuantitativo, lo cual no es de extrañar, pues la tradición de las obras en la historia económica chilena es de carácter principalmente cualitativo. No se trata de una recopilación de trabajos econométricos, pero, como lo señalan los editores en la introducción, no hay nada de qué avergonzarse. Esta obra es un gran aporte a la formación en historia económica de Chile. Para quienes desean nutrir su conocimiento sobre la materia con métodos cuantitativos, en los últimos años se han publicado varios trabajos que utilizan técnicas econométricas aplicadas, por ejemplo, al sector industrial y la antropometría; todos estos trabajos pueden encontrarse en las referencias a lo largo del manual.

En general, Historia Económica de Chile desde la Independencia presenta méritos que lo convierten en un excelente libro. Entre los aspectos más destacados se encuentran: la colaboración de autores especialistas en las temáticas tratadas; bibliografía actualizada; tópicos más demandados en la disciplina a nivel internacional (antropometría, desigualdad, entre otros); un excelente capítulo de- 
dicado a la economía mapuche, y un lenguaje académico aunque ameno. En este punto se debe hacer mención a los compiladores del libro que lograron armonizar el estilo de los contribuidores y las distintas temáticas para que luzca como un texto uniforme.

Hago una mención especial para el capítulo de Patricio Meller sobre la economía de la dictadura militar, el cual mezcla el análisis con la enseñanza de economía. A medida que el autor presenta sus argumentos también explica los conceptos y la teoría económica detrás de ellos. Si la persona que lee no entiende conceptos económicos específicos, el autor explica de forma clara los modelos e ideas que utiliza en sus planteamientos.

Uno de los puntos más destacados del manual es que los contribuidores del libro son especialistas en las materias que tratan. En historia empresarial tenemos a Ricardo Nazer, quien ha trabajado en profundidad familias empresarias como los Edwards y la familia Urmeneta, asimismo a empresas chilenas como corfo, GASco y ENDESA. Por su parte, José Díaz-Bahamonde, coautor de Chile 1810-2010. La república en cifras: Historical Statistics (2016), libro de historia económica chilena ampliamente citado, es uno de los representantes de la cliometría nacional y de los académicos que más han trabajado las políticas comerciales, y junto a Gert Wagner escribió un capítulo estilo ensayo sobre la política comercial chilena en el largo plazo. También, William Sater, autor de $A$ History of Chile, 1808-2002 (2004) junto a Simon Collier, contribuye con un capítulo sobre la industria minera como el sustento del país en el tiempo. Por otro lado, Claudio Robles, destacado académico dedicado a la historia agraria chilena, realiza una visión general sobre la transformación y el desempeño del sector agropecuario nacional. Cabe destacar a Jorge Pinto, Premio Nacional de Historia (2012), coautor junto a Luis Iván Inostroza y Manuel Llorca-Jaña del capítulo sobre la economía mapuche; tanto Jorge Pinto como Luis Iván Inostroza son los autores que más han tratado la historia económica mapuche en el último tiempo. Por último, algunos de los contribuidores (como también los editores del libro) son miembros del comité editorial de las principales revistas de investigación en historia económica dedicadas a Chile e Iberoamérica como Revista de Historia Económica, Historia Agraria, América Latina en la Historia Económica, entre otras.

Con todo, la obra presenta algunos problemas menores. Hay errores de citación de una misma obra a lo largo del texto, por ejemplo, el libro de Aníbal Pinto Santa Cruz (mencionado anteriormente), en un capítulo está citado con fecha de publicación en el año 1958 y en los demás casos en 1959 (este último es el correcto). Además, esta misma referencia es citada de dos formas distintas en la misma obra: una opción elegida fue "Pinto" y la otra "Pinto Santa Cruz", es decir, falta uniformidad en la bibliografía. Por su parte, hay algunas referencias que están escritas con "et al." sin indicar quiénes son todos los autores que participaron en la obra. En adición, algunas referencias están anotadas como"próximo"(forthcoming), omitiendo en qué volumen, número y páginas fueron publicadas, aunque han pasado más de tres años desde su publicación (revisar capítulo 19). En suma, se trata de algunos errores en criterios bibliográficos. No obstante, hay otros asuntos que merecen mayor atención.

Un problema más serio es que los capítulos "sectoriales" (sección 2 del libro) tienden a abarcar hasta la dictadura militar, descuidando el regreso a la democracia y el siglo xxi. Esto es algo que no logra suplir el trabajo sobre la economía chilena desde el regreso a la democracia (lo cual sería pedir bastante a un capítulo que ya es de alto nivel). Adicionalmente, la cobertura temporal es muy dispar entre temáticas dentro de la misma sección: algunos capítulos logran con éxito llegar hasta el "presente" (véase el capítulo de agricultura, el capítulo de empresarios y grupos económicos o el capítulo de desarrollo industrial), mientras otros finalizan incluso antes de la dictadura militar (un 
caso es el capítulo de dinero y banca). Lo anterior provoca grandes diferencias en el análisis sectorial de largo plazo. Además, queda un desequilibrio en la extensión de páginas entre un capítulo y otro. El capítulo 11 tiene más de 40 páginas y el capítulo siguiente no alcanza a tener treinta.

Un último comentario es sobre el capítulo de salarios y precios. La contribución da cuenta del comportamiento de los precios y los salarios en el largo plazo (1886-2009). Pese al aporte que realizan los autores para comprender una evolución de ambas variables económicas, este apartado no utiliza la metodología de Bare Bones Basket (BBB), popularizada por el historiador económico Robert C. Allen. Adoptar esta metodología ampliamente utilizada (basta con revisar las publicaciones de los últimos años de la Revista de Historia Económica o la revista América Latina en la Historia Económica para percatarse de su uso abundante), basada en un nivel de ingesta de calorías mínimo para sobrevivir, hubiese permitido a los autores no solo comprender la evolución de los estándares de vida de los trabajadores chilenos, sino también su comparación con otros países, especialmente de la misma región como Perú, Argentina o México, donde ya existen varios trabajos que utilizan esta metodología. Desafortunadamente, la historia económica de Chile se encuentra atrasada en este aspecto metodológico respecto a sus pares. El único trabajo que considera la metodología de Allen para Chile fue realizado por los autores del capítulo 2, "Entre la independencia y la era del salitre, c.1810-1879”, escrito por Manuel Llorca-Jaña y Juan Navarrete-Montalvo. En síntesis, el capítulo es un buen texto para estudiar salarios reales en el largo plazo, pero insuficiente para comparar con una medida "estándar" el nivel de vida de los chilenos con otros países. Cabe señalar que los mismos editores señalan la necesidad de comparar a Chile con otras economías latinoamericanas o, como indica Marcello Carmagnani en el prólogo, una comparación no solo con estas naciones, sino también con países asiáticos y europeos emergentes. Por tanto, nuevos trabajos sobre salarios reales que utilicen la metodología BBB serán un gran avance para la disciplina en Chile.

La detección de lagunas en el estudio de ciertos asuntos como un espacio para seguir construyendo historia económica es algo positivo del libro. Los autores presentan abiertamente áreas donde se puede desarrollar nueva investigación. Algunos temas son: la historia de las ciencias, la tecnología y las innovaciones, historia de la economía sumergida, historia económica regional, historia de las empresas públicas, el rol económico de la mujer, entre otros. De hecho, la mujer en este libro es apenas tratada como un análisis de brecha salarial por género y una visión del rol de la mujer dentro de la economía mapuche. Sin dudas que esto es un punto bajo, pero no es algo que se deba atribuir exclusivamente al libro, la despreocupación por el rol económico de la mujer es algo propio del desarrollo de la historiografía chilena.

Por último, resalto que la obra muestra referencias clásicas, pero también recientes en cada capítulo, junto a la identificación de fuentes primarias disponibles para trabajar. En otras palabras, el manual tiene un enfoque que promueve el desarrollo de la disciplina.

Para futuras ediciones me gustaría hacer algunas sugerencias. Hay muchos tópicos de la historia económica reciente de Chile y temas de interés de la disciplina a nivel internacional que fueron tratados somera o directamente no incluidos, y que podrían estar presentes, por ejemplo: ¿cómo afectó la crisis de la deuda externa al país?, ¿cómo se transformó la actividad económica luego del regreso a la democracia?, ¿cómo afectó el boom de los commodities a la economía local y las arcas fiscales?, ¿cómo se desarrollaron las industrias del salmón y forestal?, ¿̇cómo respondió Chile a la crisis financiera de 2007-2009?, ¿qué impacto han tenido los tratados de libre comercio en el desarrollo económico chileno?, ¿cuál es el impacto del MERCOSUR en la economía chilena? o ¿̇ómo se eliminó el problema de inflación crónica? Desafortunadamente, muy poco (o en algunos casos 
nada) de esto se verá en el libro. La falta de un examen al control de la inflación, que acompañó al país por más de un siglo (desde $c .1870$ hasta finales de la década de 1980), es un vacío importante en este manual. En suma, todos estos temas son tópicos relacionados a los años más recientes, años con altas tasas de crecimiento económico (por ejemplo, el periodo 1986-1998) y también años en que la pobreza disminuyó, la expectativa de vida aumentó y la matrícula universitaria incrementó sin precedentes.

Gran parte de la explicación para la falta de contenidos en este manual es que la disciplina está en plena expansión en Chile. Además, el periodo reciente de historia económica tiende a ser poco estudiado y la atención de la academia ha estado más cargada a unos sectores económicos por sobre otros. Por ejemplo, la industria es un sector ampliamente tratado comparado con el medio ambiente o la banca. En efecto, todavía no hay suficientes estudios sobre historia financiera y bancaria, lo que provoca que el análisis de las crisis financieras sean casos puntuales y atípicos en la historia económica chilena. Sin embargo, hay un tópico ausente en este libro que perfectamente podría estar presente: las empresas públicas.

Hay suficiente material para construir un capítulo sobre la actividad empresarial del sector público, no solo en el periodo de industrialización estimulada por el Estado, sino en el largo plazo, considerando además que hay empresas públicas que se formaron en el siglo xix. Algunas firmas que se podrían tratar son: Empresa de los Ferrocarriles del Estado (EFE), Compañía de Acero del Pacífico (CAP), Empresa Nacional del Petróleo (enAP), Compañía de Petróleos de Chile (COPEC), Líneas Aéreas Nacionales (LAN), Corporación Nacional del Cobre (CODElco), Compañía de Teléfonos de Chile (CTC), Caja de Crédito Hipotecario, Corporación de Fomento de la Producción (CORfo), Empresa Nacional de Electricidad (ENDESA), entre otras. Muchas de estas compañías, bajo el mismo o nuevos nombres comerciales, continúan operando como compañías públicas hasta el día de hoy, mientras otras fueron privatizadas. Varios contribuidores de este manual han escrito artículos o incluso libros sobre estas empresas, por lo que es factible que en futuras ediciones exista un capítulo sobre las empresas públicas chilenas.

Mi última sugerencia es más bien un deseo. Espero que esta obra dé pie para que en el futuro se publiquen más trabajos sobre Chile en disciplinas "hermanas”. Ya hay proyectos sobre la historia empresarial chilena, pero falta un libro de la evolución del pensamiento económico en Chile. Hay destacados pensadores a los cuales sería interesante visitar: Jean Gustave Courcelle-Seneuil, Marcial González, Roberto Espinoza, Ramón Santelices, Daniel Martner, la escuela relacionada a la CEPAL y los "Chicagos Boys", más recientemente, por nombrar algunos. Juan Pablo Couyoumdjian, contribuidor en este libro, es uno de los académicos que más activo ha estado en este aspecto. Otro académico relacionado al tema es José Edwards, que en 2018 publicó un capítulo sobre la evolución del pensamiento económico en el largo plazo (véase Historia del pensamiento económico en Chile, 1790-1970), una excelente síntesis de los principales contribuidores, influencias y sus aportes al desarrollo de la historia del pensamiento económico en Chile. Quizás en el mediano plazo podamos ver un libro dedicado exclusivamente a esta área y más personas involucradas en su estudio.

En resumen, esta es una excelente adición a la creciente literatura disponible sobre la historia económica chilena y está dirigida tanto a lectores académicos como al público general. Espero que se convierta en un libro de texto estándar para las y los estudiantes universitarios chilenos que se dedican a la historia y ciencias sociales afines, pero más importante en economía, ya que hasta hace poco tiempo la mayoría de los programas de licenciatura en economía tenían muy poca 
o ninguna formación en historia económica chilena. Como una de las personas a las que va dirigida la obra, agradezco la publicación de este libro y espero que aparezcan nuevas ediciones en el futuro. 